\title{
Integration of Prealbumin into Child-Pugh Classification Improves Prognosis Predicting Accuracy in HCC Patients Considering Curative Surgery
}

\author{
Xiajie Wen ${ }^{1}$, Mingjie Yao ${ }^{2}$, Yiwei Lu ${ }^{6}$, Junhui Chen ${ }^{1}$, Jiyuan Zhou ${ }^{1}$, Xiangmei Chen ${ }^{2}$, \\ Yun Zhang ${ }^{2}$, Weiquan Lu ${ }^{3}$, Xiangjun Qian ${ }^{2}$, Jingmin Zhao ${ }^{4}$, Ling Zhang* ${ }^{3}$, Shigang Ding*5 \\ and Fengmin $\mathrm{Lu}^{* 1,2}$
}

\begin{abstract}
${ }^{1}$ Department of Minimally Invasive Intervention, Peking University Shenzhen Hospital, Shenzhen PKU-HKUST Medical Center, Shenzhen, China; ${ }^{2}$ Department of Microbiology \& Infectious Disease Center, School of Basic Medical Sciences, Peking University Health Science Center, Beijing, China; ${ }^{3}$ Department of Hepatopancreatobiliary Surgery, Henan Cancer Hospital Affiliated to Zhengzhou University, Zhengzhou, China; ${ }^{4}$ Department of Pathology and Hepatology, Beijing 302 Hospital, Beijing, China;

${ }^{5}$ Peking University Third Hospital, Department of Gastroenterology, Beijing, China; 6 University of North Carolina - Eshelman School of Pharmacy, Chapel Hill, NC, USA
\end{abstract}

\begin{abstract}
Background and Aims: The poor outcomes of hepatocellular carcinoma (HCC) patients may be due to not only malignant tumors but also limited liver function. Therefore, as stated in major guidelines, only patients with relatively normal liver function (Child-Pugh A) would be referred for curative hepatectomy. Even so, the postsurgery survival rate of patients is still extremely poor. Direct curative resection may benefit most patients. This study aimed to improve the prognosis predicting accuracy of the Child-Pugh scoring system. Methods: This study included two cohorts: cohort A being composed of 613 HCC patients, with a 23-month median postsurgery follow-up time; and cohort B being composed of 554 tumor-free chronic liver disease patients. Kaplan-Meier test and Cox model were used for survival analysis. Independent-samples $t$ test or one-way ANOVA was used to test the differences between different groups. Results: Serum prealbumin levels were found inversely correlated with worsening of fibrotic scores $(r=-0.482, p<0.001)$. Lower levels of presurgery prealbumin was an independent factor of poor postsurgery prognosis in Child-Pugh A patients, with a hazard ratio of 0.731 ( $p=$ 0.001 ). By integrating prealbumin together with total bilirubin level, serum albumin concentration and prothrombin time, a modified liver disease prognosis scoring system was developed to define traditional Child-Pugh A HCC patients as Modified Child-Pugh MCP-1, MCP-2 and MCP-3, with median postsurgery
\end{abstract}

Keywords: Hepatocellular carcinoma; Curative resection; Survival; Child-Pugh prealbumin.

Abbreviations: AFP, alpha fetoprotein; Alb, albumin; AST, aspartate transaminase; BCLC, Barcelona Clinic Liver Cancer; CLD, chronic liver disease; GGT, gamma-glutamyl transferase; HCC, hepatocellular carcinoma; MELD, model for end-stage liver disease; PA, prealbumin; PT, prothrombin time; TBIL, total bilirubin. Received: 8 January 2018; Revised: 6 April 2018; Accepted: 17 May 2018

* Correspondence to: Fengmin Lu, Department of Microbiology \& Infectious Disease Center, School of Basic Medical Science, Peking University Health Science Center, Beijing 100191, China. Tel: +86-10-8280-5136, Fax: +86-10-8280-5136, E-mail: lu.fengmin@hsc.pku.edu.cn; Shigang Ding, Department of Gastroenterology, Peking University Third Hospital, Beijing 100191, China. Tel: +8615611908241, Fax: +86-10-62017700, E-mail: dingshigang222@163.com; Ling Zhang, Department of Hepatopancreatobiliary Surgery, Henan Cancer Hospital Affiliated to Zhengzhou University, Zhengzhou 450001, China. Tel: +86-3716587787, Fax: +86-371-6587787, E-mail: zhanglily561@126.com overall survival times of $44.00,28.00$ and 11.00 months respectively. Conclusions: Preoperative serum prealbumin is a valuable prognosis predicting biomarker for Child-Pugh A HCC patients who may be under consideration for curative resection. With serum prealbumin included as one of the parameters, the MCP scoring system might improve the postsurgery survival predicting accuracy for HCC patients.

Citation of this article: Wen X, Yao M, Lu Y, Chen J, Zhou J, Chen $X$, et al. Integration of prealbumin into Child-Pugh classification improves prognosis predicting accuracy in HCC patients considering curative surgery. J Clin Transl Hepatol 2018;6(4):377-384. doi: 10.14218/JCTH.2018.00004.

\section{Introduction}

Primary liver cancer is one of the most lethal human malignancies worldwide. ${ }^{1}$ China alone accounts for about $50 \%$ of the annual total number of new cases and deaths. Among them, $85-90 \%$ are hepatocellular carcinoma (HCC)-related cases. ${ }^{2}$ Although there have been some improvements in diagnosis and treatment, the mortality rate of HCC remains unacceptably high and long-term outcomes remain suboptimal. ${ }^{3,4}$

Poor postsurgery outcomes have been mainly attributed to tumor-related factors, including tumor size, vascular invasion, p53 mutation, and higher alpha fetoprotein (AFP), etc. ${ }^{5-10}$ The liver is a vital organ of the human body. Its wide range of functions include protein synthesis, detoxification of various metabolites, production of hormones, production of biochemicals necessary for digestion, etc. Since a majority of liver cancer patients also suffer from chronic liver disease (CLD), cirrhosis is very common among patients with liver cancer. ${ }^{2}$ Therefore, it is reasonable to postulate that the limited functions of the liver postresection may greatly affect the prognosis of patients who undergo curative surgical treatment.

Currently, though several treatment choices are available to HCC patients, liver resection remains the only treatment with curative potential. Thus, DC resection may benefit most patients, the newly updated American Association for the Study of Liver Diseases guideline for the treatment of HCC (2017) recommends that only Child-Pugh A patients, in which liver function is considered to be relatively close-to-normal, be referred to 
curative surgery. ${ }^{11}$ The Child-Pugh classification system was developed in 1973 to estimate the risk of operative mortality in patients with bleeding esophageal varices. ${ }^{12}$ Since then it has been modified, refined, and become a widely used tool to assess prognosis in patients with CLD and cirrhosis. ${ }^{13}$

In addition to the two factors based on clinical assessment (i.e. degree of ascites and degree of hepatic encephalopathy), the other three parameters for scoring (i.e. total bilirubin (TBIL), serum albumin (Alb), and prothrombin time (PT)) are indicators of the synthetic function of the liver. Prealbumin (PA) is a recently available biomarker indicative of liver synthetic functions and was not included in the traditional Child-Pugh classification system. In this study, the prognosis predicting value of PA on the long-term postsurgery survival of HCC patients who underwent hepatic resection was evaluated. More than that, by integrating preoperative PA as a parameter along with the three traditional parameters of TBIL, Alb, and PT, a new classification system was developed with improved prognosis predicting accuracy in Child-Pugh A HCC patients planning to undergo curative resection.

\section{Methods}

\section{Study population and clinical data collection}

This study included two cohorts. Cohort A was composed of 613 Chinese HCC patients who underwent curative resection in Henan Cancer Hospital (Zhengzhou, China) between February 2009 and July 2013. The inclusion criteria for the patients
Wen X. et al: Prealbumin can be used as prognosis factor

were: (1) HCC diagnosis confirmed by pathology; (2) no receipt of chemotherapy or radiotherapy before the surgery; and (3) adequate clinical data that could be checked in the hospital. All patients were enrolled for follow-up. The follow-up was carried out every 3 months in the first year and every 6 months in the next 4 years. The last follow-up time was January 2017. During the follow-up process, $75(12.2 \%)$ patients were lost.

In order to investigate the correlation between liver synthetic function-relevant biomarkers, including PA to patient liver fibrosis/cirrhosis severity, a cross-sectional study was carried out using cohort $\mathrm{B}$, which was composed of 554 tumor-free precirrhosis and cirrhosis patients with CLD. The fibrosis degree was assessed according to the Metavir scoring system. Fibrosis was staged on a 0-4 scale: F0, no fibrosis; F1, portal fibrosis without septa; F2, portal fibrosis and a few septa extending into lobules; F3, numerous septa extending to adjacent portal tracts or terminal hepatic venules; and F4, cirrhosis. ${ }^{14}$

This study was approved by the Ethics Committee of Peking University Health Science Center (Beijing, China). Written informed consent was obtained from all participants.

\section{Statistical analysis}

Cumulative survival was assessed by the Kaplan-Meier test. The differences of survival curves were analyzed using logrank analysis. Cox modeling was performed to assess whether the biomarkers of liver synthetic functions were independent factors to patients' postsurgery overall survival. Correlations between the three biomarkers and basic clinical

Table 1. Characteristics of the patients

\begin{tabular}{|c|c|c|c|c|}
\hline \multirow[b]{2}{*}{ Variable } & \multicolumn{2}{|c|}{ HCC patients $(n=613)$} & \multicolumn{2}{|c|}{ CLD patients $(n=554)$} \\
\hline & $\mathrm{N}$ & Mean \pm SD & $\mathrm{N}$ & Mean \pm SD \\
\hline Sex, M/F & $502 / 111$ & - & $305 / 249$ & - \\
\hline Fibrosis stage, F0-1/F2/F3/F4 & - & - & $198 / 96 / 85 / 175$ & - \\
\hline $\mathrm{HBV},+/-$ & $485 / 119$ & - & $220 / 309$ & - \\
\hline $\mathrm{HCV},+/-$ & $19 / 594$ & - & $46 / 508$ & - \\
\hline $\mathrm{BCLC}, \mathrm{A} / \mathrm{B}$ & $514 / 99$ & - & - & - \\
\hline Child-Pugh, A/B/C & $545 / 68 / 0$ & - & $402 / 52 / 6$ & - \\
\hline Age, $<60 / \geq 60$ years & $475 / 138$ & $52.94 \pm 10.41$ & $491 / 63$ & $43.67 \pm 12.47$ \\
\hline Tumor size, $<5 / \geq 5 \mathrm{~cm}$ & $207 / 403$ & $8.51 \pm 5.32$ & - & - \\
\hline $\mathrm{GGT}, \leq 50 />50 \mathrm{U} / \mathrm{L}$ & $188 / 423$ & $122.73 \pm 127.28$ & $272 / 277$ & $107.84 \pm 178.82$ \\
\hline $\mathrm{ALT}, \leq 40 />40 \mathrm{U} / \mathrm{L}$ & $321 / 290$ & $64.66 \pm 132.96$ & $256 / 293$ & $118.80 \pm 221.85$ \\
\hline $\mathrm{AST}, \leq 40 />40 \mathrm{U} / \mathrm{L}$ & $209 / 402$ & $71.69 \pm 136.70$ & $298 / 251$ & $83.93 \pm 165.20$ \\
\hline $\mathrm{ALP}, \leq 150 />150 \mathrm{U} / \mathrm{L}$ & $496 / 115$ & $118.32 \pm 70.64$ & $457 / 92$ & $116.13 \pm 98.96$ \\
\hline Alb, $<35 / \geq 35 \mathrm{~g} / \mathrm{L}$ & $67 / 544$ & $40.53 \pm 5.37$ & $82 / 467$ & $39.40 \pm 4.75$ \\
\hline $\mathrm{PA},<200 / \geq 200 \mathrm{mg} / \mathrm{L}$ & $420 / 104$ & $155.83 \pm 53.81$ & $334 / 214$ & $175.88 \pm 69.52$ \\
\hline Platelet count, $<100 / 100-300 />300 \times 10^{9} / \mathrm{L}$ & $137 / 433 / 37$ & $158.38 \pm 78.82$ & $92 / 442 / 16$ & $169.22 \pm 68.33$ \\
\hline $\mathrm{PT}, \leq 14.3 />14.3 \mathrm{~s}$ & $460 / 139$ & $13.35 \pm 2.08$ & $250 / 301$ & $11.35 \pm 1.41$ \\
\hline $\mathrm{RBC},<3.5 / \geq 3.5 \times 10^{12} / \mathrm{L}$ & $62 / 510$ & $4.24 \pm 0.68$ & $207 / 343$ & $4.45 \pm 0.63$ \\
\hline $\mathrm{AFP},<400 / \geq 400 \mathrm{ng} / \mu \mathrm{L}$ & $338 / 268$ & $513.15 \pm 536.29$ & $550 / 4$ & $15.28 \pm 71.53$ \\
\hline TBIL,$\leq 17.1 />17.1 \mu \mathrm{mol} / \mathrm{L}$ & $402 / 209$ & $17.55 \pm 19.70$ & $370 / 179$ & $25.36 \pm 43.64$ \\
\hline
\end{tabular}

Abbreviations: Alb, albumin; AFP, alpha fetoprotein; ALP, alkaline phosphatase; ALT, alanine aminotransferase; AST, aspartate transaminase; BCLC, Barcelona Clinic Liver Cancer; GGT, gamma-glutamyl transferase; HBV, hepatitis B virus; HCV, hepatitis C virus; PA, prealbumin; PT, prothrombin time; RBC, red blood cell; TBIL, total bilirubin. 
data were analyzed using Spearman's correlation. As for quantitative indicators, independent samples $t$-test or oneway ANOVA were used to test the difference between different groups. Two-sided $p$-values less than 0.05 were defined as statistically significant. All analyses were performed with SPSS v21.0 (Xishu Software Company, Shanghai, China). The cut-off value in this research was found through receiver operating characteristic curves. ${ }^{15}$

\section{Results}

\section{Characteristics and clinical data of patients}

This study enrolled two cohorts, including $613 \mathrm{HCC}$ patients for cohort A and 554 CLD patients for cohort B. For cohort A, the median follow-up time was 23 months. The normal reference levels of the biochemistry variables were those used in the hospital. In addition, Child-Pugh classes $A$ to $C$ were determined by the following 5 assessments: concentrations of TBIL, Alb and PT, and degrees of ascites and hepatic encephalopathy; the Barcelona Clinic Liver Cancer (BCLC) stages A to D were determined by Child-Pugh classification, performance

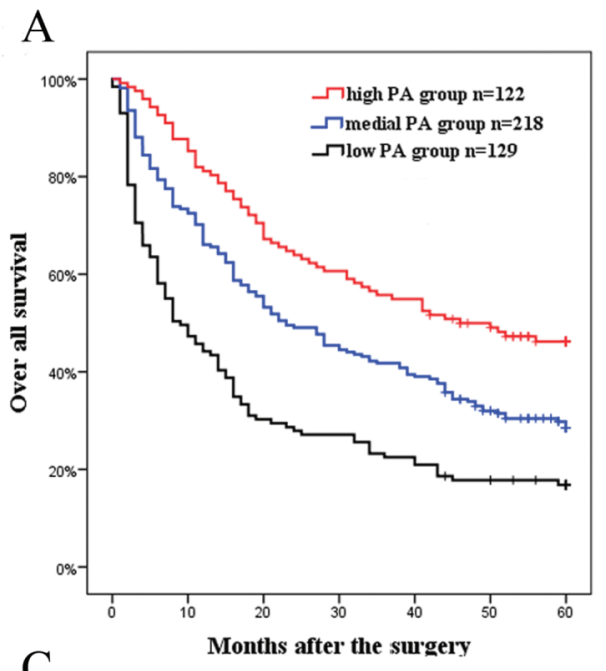

C

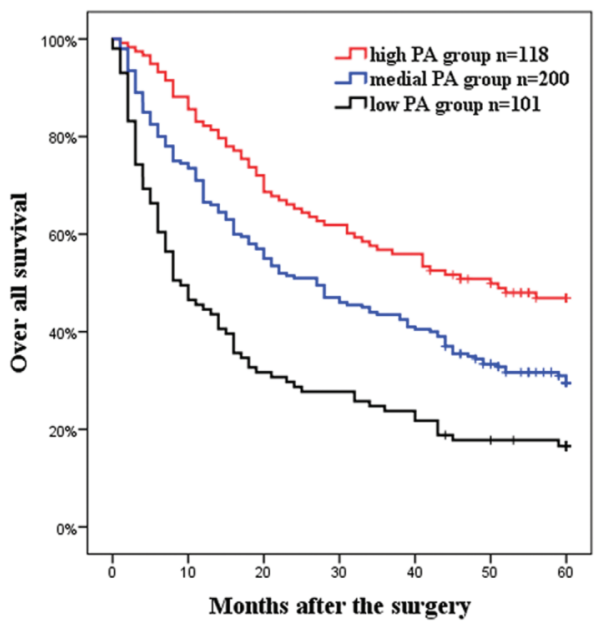

status, and tumor status. The baseline characteristics of the patients are shown in Table 1.

\section{Presurgery PA was an independent prognosis factor for Child-Pugh A HCC patients who underwent curative surgery}

First, the differences in the level of PA and other parameters, including PT prolonged second, TBIL and Alb, between patients with different background were analyzed. Of the four parameters, PA exhibited significant differences with obvious changes in value in regards to patient sex, chronic hepatitis $B$ or $\mathrm{C}$ virus infection, the stage of BCLC classification, and ChildPugh scores (Supplementary Table 1).

Since male patients had higher PA levels than that of females, sex differences were taken into consideration when trying to set the optimized cut-off values via receiver operating characteristic curve plotting. Ranges of "normal" PA levels were derived for male and female patients, being $120-190 \mathrm{mg} / \mathrm{L}$ and $110-170 \mathrm{mg} / \mathrm{L}$ respectively, which was different from the normal range of $200-400 \mathrm{mg} / \mathrm{L}$ referred for both male and female individuals in current clinical practices.

\section{B}
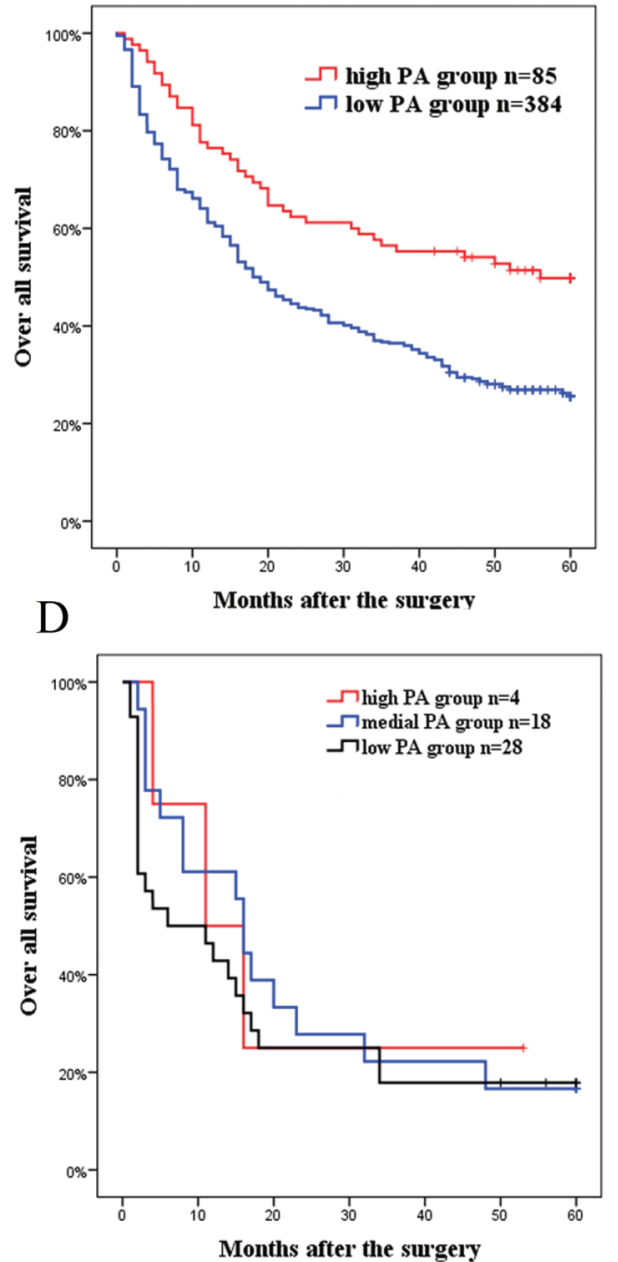

Fig. 1. Kaplan-Meier curves for different PA group patients. (A) Best cut-off value (low PA group: male $<120 \mathrm{mg} / \mathrm{L}$, female $<110$; medium $P A$ group: male $120-190 \mathrm{mg} / \mathrm{L}$, female $110-170 \mathrm{mg} / \mathrm{L}$; high PA group: male $>190 \mathrm{mg} / \mathrm{L}$, female $>170 \mathrm{mg} / \mathrm{L})(p<0.001)$. (B) Clinical cut-off value (low PA group: $<200 \mathrm{mg} / \mathrm{L} ;$ high PA group: $\geq 200 \mathrm{mg} / \mathrm{L}$ ) $(p<0.001)$. (C) Child-Pugh A patients divided according to the best cut-off of PA $(p<0.001)$. (D) Child-Pugh B patients divided according to the best cut-off of PA ( $p=0.719)$. 
Wen X. et al: Prealbumin can be used as prognosis factor

Next, the patients were subdivided into three groups according to the new cut-off values for male and female, including low PA, medium PA, and high PA groups. As expected, patients in the high PA group exhibited the highest postsurgery survival rate, while patients in the low PA group had the lowest survival rate, as shown by the survival curve analysis (Fig. 1A). No patients in this cohort had preoperative serum PA higher than $400 \mathrm{mg} / \mathrm{L}$, so based on the clinical cut-off value, the patients can only be divided into two groups. As shown in Fig. 1B, the same tendency was also observed when the patients were divided according to the clinical cutoff value $(200 \mathrm{mg} / \mathrm{L})$. Interestingly, further stratified analyses revealed that PA exhibited prognosis predicting power only in Child-Pugh A HCC patients $(p<0.001$; Fig. 1C) and not in Child-Pugh B patients ( $p=0.719$; Fig. 1D).

The new American Association for the Study of Liver Diseases guide for the treatment of HCC (2017) does not refer to curative resection if a patient's tumor size is $\geq 5 \mathrm{~cm},{ }^{11}$ though some patients may still be referred to surgery in China. ${ }^{16}$ Next, according to their tumor size, the Child-Pugh A patients were further divided into the big-tumor group $(\geq 5 \mathrm{~cm})$ and small-tumor group $(<5 \mathrm{~cm})$. Then, the prognosis predicting role of PA was evaluated respectively in the two groups. As shown in Supplementary Fig. 1, among the Child-Pugh A patients, the PA-high individuals had longer postsurgery survival time than PA-low individuals, both in those patients harboring small tumors (median overall survival times: $>60$ months vs. 19 months, $p<0.001$ ) as well as in those patients with large tumors (median overall survival times: 28 months vs. 10 months, $p<0.001$ ). Noticeably, in patients classified as Child-Pugh $A$, the median overall survival times of those patients with small tumor size was much better than those with tumors $\geq 5 \mathrm{~cm}$ ( $>60$ months vs. 28 months, $p<$ 0.001 ). However, presurgery PA level failed to act as a prognosis predicting marker in Child-Pugh B patients $(p=0.162)$.

To further confirm the prognosis predicting power of presurgery PA level using Cox regression, a total of 14 potential clinical variables were analyzed as candidate factors associated with Child-Pugh A HCC patients' prognosis. As shown in Table 2, tumor size, BCLC classification, the levels of AFP, aspartate transaminase (AST) and gamma-glutamyl transferase (GGT), and platelet count were successfully isolated as independent predictors for patients' overall postsurgery survival, giving hazard ratio values of $1.605,1.441,1.366,1.502,1.419$ and 0.771 , respectively. In addition to these already known factors, PA was also identified as an independent predictor. With a hazard ratio value of 0.731 , preoperative $\mathrm{PA}$ level was a protective factor for patient overall survival in Child-Pugh A HCC patients.

\section{PA levels were inversely correlated with worsening fibrotic/cirrhotic scores among the tumor-free CLD patients}

The synthetic function of the liver is significantly influenced by CLD progression, particularly fibrosis/cirrhosis. To find the relationship between fibrotic severity and PA levels in CLD patients, a cohort of 554 tumor-free CLD patients was used. At least two pathologists scored each patient's degree of fibrosis independently. In these patients, all five parameters (PT, TBIL, Alb, PA, AST, and GGT) showed gradual but significant changes in concentration accompanied by an increase in fibrotic scores (from F0-1 to F2, F3 and F4) (Table 3).

Regarding PA, the correlation coefficients ( $r$-values) between the decline of serum PA level and the worsening of fibrotic scores was $-0.482(p<0.001)$ overall, $-0.420(p<0.001)$ for hepatitis B virus-positive patients, $-0.485(p=0.002)$ for hepatitis $C$ virus-positive patients, $-0.308(p=0.002)$ for nonalcoholic steatohepatitis patients, and $-0.454(p<0.001)$ for other patients. Compared to AST and GGT, which have been regarded as the indicators of liver fibrotic severity, serum PA had a relatively small individual difference in the patients with the same fibrotic scores, while the relative larger standard

Table 2. Multivariate Cox proportional hazard model for predictors of death for Child-Pugh A patients

\begin{tabular}{|c|c|c|c|c|}
\hline Variable & $\beta$ & $\mathrm{HR}$ & $95 \% \mathrm{CI}$ & $P$ \\
\hline Sex, M/F & -0.192 & 0.825 & $0.588,1.157$ & 0.265 \\
\hline Age, $<60 / \geq 60$ years & 0.058 & 1.060 & $0.779,1.441$ & 0.712 \\
\hline Tumor size, $<5 / \geq 5 \mathrm{~cm}$ & 0.473 & 1.605 & $1.181,2.181$ & 0.003 \\
\hline $\mathrm{BCLC}, \mathrm{A} / \mathrm{B}$ & 0.365 & 1.441 & $1.066,1.947$ & 0.017 \\
\hline $\mathrm{AFP},<400 / \geq 400 \mathrm{ng} / \mu \mathrm{L}$ & 0.312 & 1.366 & $1.065,1.752$ & 0.014 \\
\hline $\mathrm{ALT}, \leq 40 />40 \mathrm{U} / \mathrm{L}$ & -0.207 & 0.813 & $0.616,1.072$ & 0.142 \\
\hline AST, $\leq 35 />35 \mathrm{U} / \mathrm{L}$ & 0.407 & 1.502 & $1.103,2.046$ & 0.010 \\
\hline$A L P, \leq 150 />150 \mathrm{U} / \mathrm{L}$ & 0.247 & 1.280 & $0.943,1.738$ & 0.113 \\
\hline $\mathrm{GGT}, \leq 50 />50 \mathrm{U} / \mathrm{L}$ & 0.350 & 1.419 & $1.032,1.950$ & 0.031 \\
\hline TBIL,$\leq 17.1 />17.1 \mu \mathrm{mol} / \mathrm{L}$ & 0.011 & 1.011 & $0.776,1.319$ & 0.933 \\
\hline $\begin{array}{l}\text { Platelet count, } \\
<100 / 100-300 />300 \times 10^{9} / \mathrm{L}\end{array}$ & -0.319 & 0.771 & $0.566,0.933$ & 0.012 \\
\hline $\mathrm{PT},<12 / 12-14 />14 \mathrm{~s}$ & 0.082 & 1.086 & $0.795,1.482$ & 0.604 \\
\hline Alb,$<34 / 34-48 />48 \mathrm{~g} / \mathrm{L}$ & -0.246 & 0.782 & $0.501,1.218$ & 0.277 \\
\hline $\begin{array}{l}\mathrm{PA} \text {, male }<120 / 120-190 />190 \mathrm{mg} / \mathrm{L} \text {, } \\
\text { female }<110 / 110-170 />170 \mathrm{mg} / \mathrm{L}\end{array}$ & -0.313 & 0.731 & $0.606,0.883$ & 0.001 \\
\hline
\end{tabular}

Abbreviations: Alb, albumin; AFP, alpha fetoprotein; ALP, alkaline phosphatase; ALT, alanine aminotransferase; AST, aspartate transaminase; BCLC, Barcelona Clinic Liver Cancer; CI, confidence interval; GGT, gamma-glutamyl transferase; HR, hazard ratio; PA, prealbumin; PT, prothrombin time; TBIL, total bilirubin. 


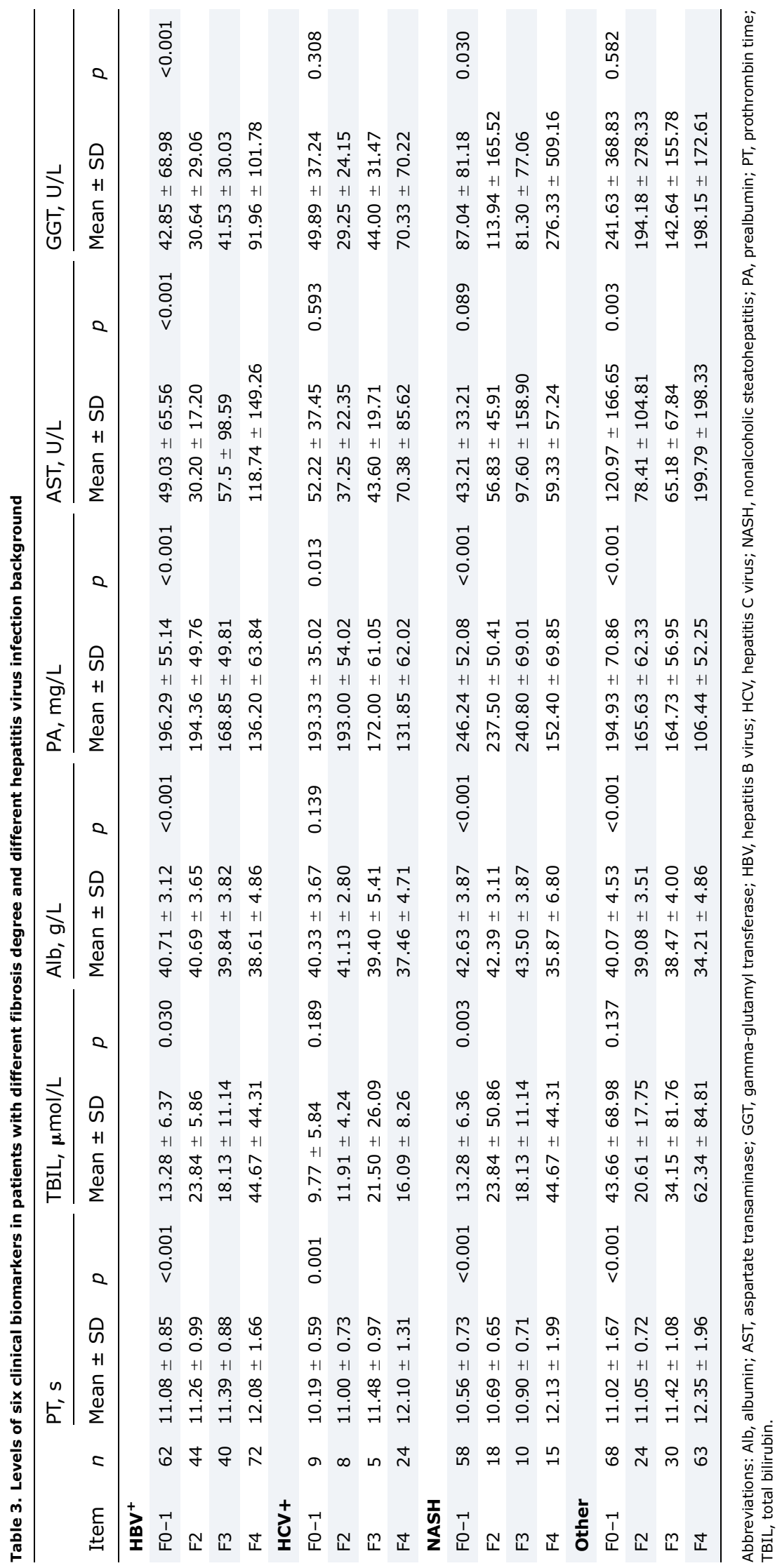


Wen X. et al: Prealbumin can be used as prognosis factor

deviation of AST and GGT indicated that they could be affected by other factors, for example, necroinflammation of the liver.

The data here implicate the potential use of serum PA as a surrogate marker functionally reflecting fibrosis severity. However, PA did not change a lot between patients with fibrosis stage F1-F2, so the use of PA in diagnosing early fibrosis would be limited.

\section{Pre-surgery PA levels were able to further subdivide Child-Pugh class A HCC patients with significantly different postsurgery overall survival times}

Since preoperative PA level was found to greatly influence HCC patients' prognosis, we wondered if PA might improve the accuracy of the Child-Pugh system in predicting postsurgery survival among HCC patients. Therefore, based on presurgery PA levels, the Child-Pugh A patients were further subgrouped into PA-high, PA-medium and PA-low groups. As expected, the median overall postsurgery survival times varied greatly, from more than 49 months to as short as 11 months among those Child-Pugh A patients (for details, see Table 4). In contrast, such significant differences were not observed among the Child-Pugh B patients, largely because the latter always had extremely poor overall survival.

By adding presurgery serum PA as a new parameter, the traditional Child-Pugh classification was modified (Table 5). With different ranges between male and female patients, scores of 1,2 or 3 were given to patients classified as PAhigh, PA-medium, and PA-low, respectively. Then, the differences of Kaplan-Meier survival curves between Child-Pugh classification and the modified Child-Pugh (MCP) classifications were analyzed. As shown in Fig. 2 and Table 6, the classic Child-Pugh scoring system classified the patients into class $A$ and class $B$ as expected, with the median survival times being 27 months and 11 months, respectively. In contrast, the MCP classification system was able to divide the patients into groups named as MCP-1 (score $=4)$, MCP-2 (score $=5$ ) and MCP-3 (score $\geq 6$ ), with median survival times at 44 months, 28 months and 11 months, respectively. This result indicated that the MCP classification system exhibited a much higher accuracy in prognosis prediction (Table 6).

From the survival curves based on presurgery PA level, it appeared as if PA represented the main factor for prognosis prediction in this system (Fig. 1). Only HCC patients of ChildPugh classification A with tumor size less than $5 \mathrm{~cm}$ are recommend for liver resection in the newly updated guidelines; ${ }^{11}$ accordingly, the Child-Pugh A patients in cohort A were subgrouped into small tumor size $(<5 \mathrm{~cm})$ and larger tumor size $(\geq 5 \mathrm{~cm})$ groups. As expected, in the small tumor size patient group, the median survival times were more than 60 months, 52 months and 6 months respectively for stage MCP-1, MCP-2

Table 4. Association of pre-surgery PA levels and the postsurgery overall survival among the Child-Pugh class $A$ and $B$ patients

\begin{tabular}{llll}
\hline Child-Pugh & $\begin{array}{l}\text { Low PA } \\
\text { group }\end{array}$ & $\begin{array}{l}\text { Medium PA } \\
\text { group }\end{array}$ & $\begin{array}{l}\text { High PA } \\
\text { group }\end{array}$ \\
\hline A & $101(11.02)$ & $200(26.25)$ & $118(49.85)^{*}$ \\
B & $28(11.20)$ & $18(16.00)$ & $4(12.00)^{* *}$ \\
\hline
\end{tabular}

Data are presented as $n$ (median survival time in months. ${ }^{*} p<0.001 ;{ }^{* *} p=$ 0.719 .

Abbreviation: PA, prealbumin
Table 5. New classification scores of the MCP system

\begin{tabular}{llll}
\hline & \multicolumn{3}{c}{ Score } \\
\cline { 2 - 4 } Item & 1 & 2 & 3 \\
\hline Bilirubin, $\mu \mathrm{mol} / \mathrm{L}$ & $<34$ & $34 \sim 51$ & $>51$ \\
$\begin{array}{l}\text { Alb, g/L } \\
\begin{array}{l}\text { Prothrombin time } \\
\text { prolongation, s }\end{array}\end{array}$ & $>35$ & $28 \sim 35$ & $<28$ \\
$\begin{array}{l}\text { Preoperative PA for } \\
\text { males, mg/L }\end{array}$ & $>190$ & $120 \sim 190$ & $<6$ \\
$\begin{array}{l}\text { Preoperative PA for } \\
\text { females, mg/L }\end{array}$ & $>170$ & $110 \sim 170$ & $<110$ \\
\hline
\end{tabular}

MCP-1 (score $=4)$, MCP-2 $($ score $=5)$, MCP-3 $($ score $\geq 6)$.

Abbreviations: Alb, albumin; MCP, modified Child-Pugh; PA, prealbumin.

and MCP-3 patients. In contrast, the corresponding median survival times for the patients with larger tumors were 27 months, 20 months and 9 months respectively. Concordantly, the 1-, 3- and 5-year postsurgery survival rates showed the same tendencies (see Supplemental Table 2).

\section{Discussion}

In the past decade, several treatment options have become available for the management of HCC. Among them, hepatectomy remains the therapy with the highest likelihood for longterm cure potential. However, postsurgery survival has varied greatly for those patients with indication of curative liver resection. Because a majority of the HCC patients simultaneously suffer from cirrhosis, among the factors influencing the postsurgery survival the remaining liver function is a strong determinant.

Currently there are several models available to evaluate the patients' remaining liver function; among them, Child-Pugh score and model for end-stage liver disease (MELD) are the two most widely used classification systems. ${ }^{17,18}$ The MELD scoring system was originally developed based on survival data from patients who underwent elective transjugular intrahepatic portosystemic shunt procedure, and now it is recommended to estimate the survival probability of a patient with end-stage liver disease. Therefore, the MELD scoring system was excluded because a patient with end-stage liver disease may not survive the surgical operation itself. The Child-Pugh scoring system is usually used to assess the prognosis of CLD (mainly cirrhosis), and also assist in preselection of patients. The majority of HCC management guidelines recommend that only Child-Pugh A patients be recommend for curative resection. Of the five parameters which composed the Child-Pugh classification system, ascites and encephalopathy are two subjective ones that are strongly associated with decomposition and were, therefore, used as an exclusion category for hepatectomy. The other three parameters, bilirubin, albumin and prothrombin time, all reflect the synthetic function of the liver. Among them, serum albumin was the most prominent prognostic indicator of survival in liver cirrhosis. ${ }^{19,20}$ PA, a small molecule protein synthesized by liver cells, is now also considered to be a sensitive biomarker of liver synthesis function, but has not been used as a parameter in the Child-Pugh scoring system before. Compared to albumin's 19-21 days-long half-life, the 
Wen X. et al: Prealbumin can be used as prognosis factor
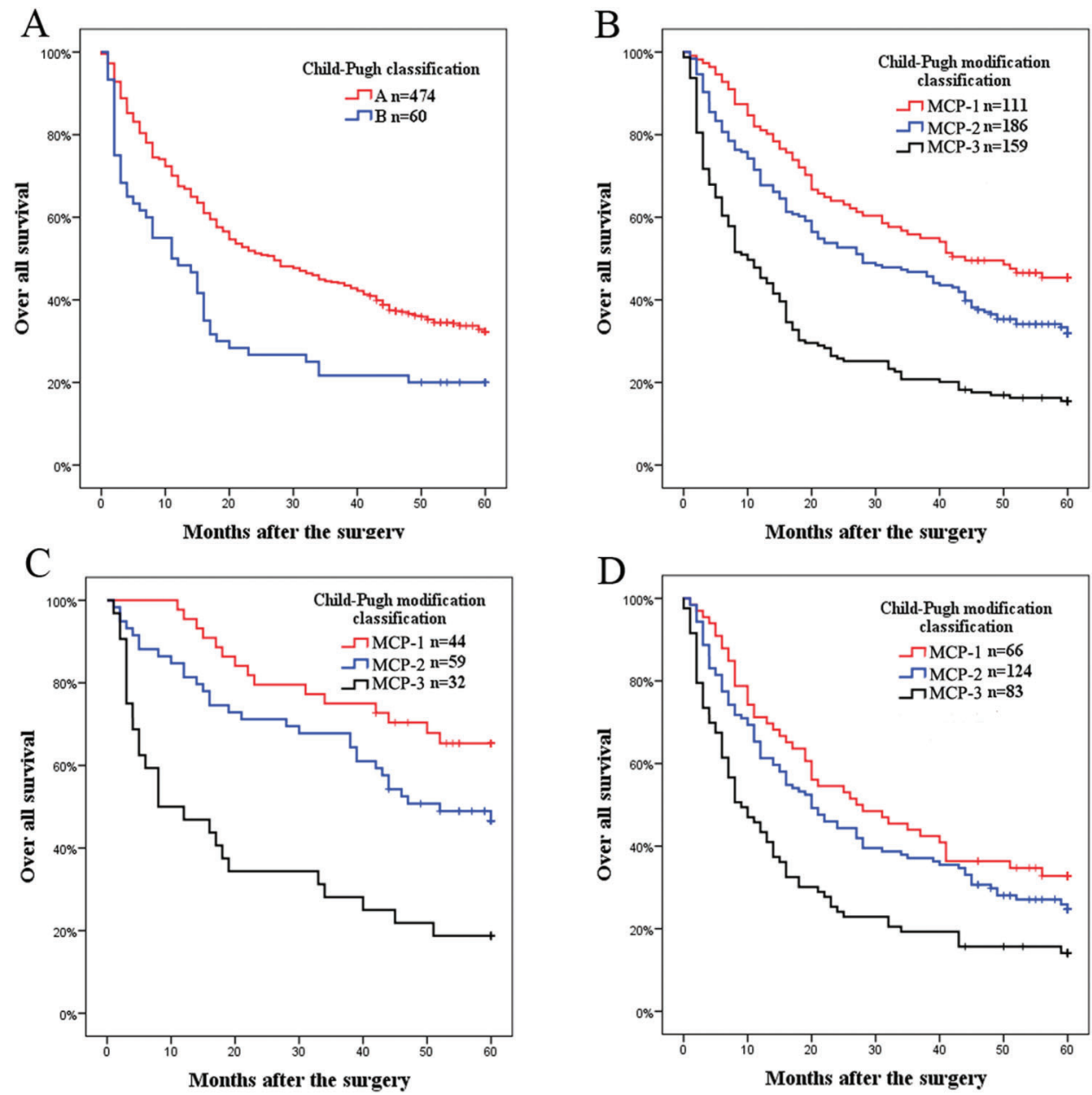

Fig. 2. Kaplan-Meier curves for Child-Pugh classification and modified Child-Pugh classification. (A) Patients were divided according to the Child-Pugh score $(p<$ 0.001). (B) Patients were divided according to the modified Child-Pugh classification $(p<0.001)$. (C) Child-Pugh A patients with small tumor size $(<5 \mathrm{~cm})(p<0.001)$. (D) Child-Pugh A patients with larger tumor size $(\geq 5 \mathrm{~cm})(p<0.001)$

half-life of PA is as short as 1.9 days. ${ }^{21}$ Such characteristics make PA a much more sensitive biomarker of liver function.

In this prospective study, we first demonstrated that presurgery PA is a prognosis factor for postsurgery ChildPugh A HCC patients, which could be used to stratify the patients as PA-high, PA-medium and PA-low groups with distinct postsurgery overall survival times. In line with this, multifactorial survival analysis showed that PA was an independent predictive factor of Child-Pugh A HCC patients with liver resection, in addition to tumor size and other well-known prognostic factors, such as AFP and GGT. These results were partly consistent with the results reported by Zhao et al. ${ }^{22}$ Though blood platelet count was also an independent prognosis factor of HCC patients, in the following analysis, platelet count was not considered because it is not a factor that directly reflects liver function and it can be affected by many factors, such as a blood transfusion and splenectomy. Finally, a MCP scoring system with bilirubin, Alb, PT prolongation and PA as parameters was developed specifically for the selection of appropriate HCC patients for curative resection.

Table 6. Child-Pugh compared to MCP classification system

\begin{tabular}{lllll}
\hline & \multicolumn{3}{c}{ Child-Pugh classification } & Median survival time in months \\
\cline { 2 - 4 } & & $\mathrm{A}, n$ & $\mathrm{~B}, n$ & 44.00 \\
\hline MCP classification & $\mathrm{MCP}-1$ & 131 & 0 & 28.00 \\
& $\mathrm{MCP}-2$ & 200 & 1 & 11.00 \\
Median survival time in months & $\mathrm{MCP}-3$ & 133 & 56 & 11.00 \\
\hline
\end{tabular}

Abbreviation: MCP, modified Child-Pugh. 
Wen X. et al: Prealbumin can be used as prognosis factor

According to the newly updated American Association for the Study of Liver Diseases guideline for the treatment of HCC (2017), HCC patients who have Child-Pugh A cirrhosis and resectable T1 or T2 (tumor size less than $5 \mathrm{~cm}$ ) should choose resection over radiofrequency ablation to improve their chances of a longer survival time. ${ }^{11}$ Even so, median postsurgery survival times still varied largely among the patients scored by the Child-Pugh classification system. For example, after excluding patients with tumor size larger than $5 \mathrm{~cm}$, 35 patients in this study met the criteria of American Association for the Study of Liver Diseases guidelines but were classified into the MCP-3 group, with a median survival time of only 6 months. These results indicate that the MCP classification system can sensitively exclude those patients from curative liver resection, even though they previously had no contraindication for surgery. Thus, we suggest that HCC patients classified as MCP-1 and MCP- 2 would benefit most from curative surgery, while hepatectomy classified as MCP-3 should be reconsidered by doctors.

Accumulating clinical data indicates that liver fibrosis is not only relevant to liver functional reserve but also intertwined with the prognosis of $\mathrm{HCC}^{3}$ In line with this, in this crosssectional study we observed an obvious correlation between PA concentration and the degree of fibrosis/cirrhosis stages $(r=-0.482, p<0.001)$. These results may explain in part why PA acts as a strong biomarker of liver function.

There are some questions that need to be addressed in the future. First, serum PA concentration may be affected by other diseases, such as obstructive jaundice, hyperthyroidism, nephritic syndrome, and ulcerative colitis. Secondly, nutritional condition can greatly affect an individual's serum level of PA. For example, it was much higher in nonalcoholic steatohepatitis patients in this study, so the cut-off value may vary according to the etiology of a patient.

In conclusion, presurgery PA levels could be a valuable predictor of postsurgery survival of HCC patients who undergo curative liver resection. Integrating PA level into this new MCP classification system may improve the postsurgery survival predicting accuracy of HCC patients. We suggest that HCC patients scored as MCP-1 and MCP-2 would benefit the most from curative liver resection, especially those who have no other surgical contraindications.

\section{Acknowledgments}

This work was supported by the SZSM201612071, the National S \& T Major Project for Infectious Diseases (2017YFC0908100, 2017ZX10302201, 2017ZX10201201) and the project from Beijing Municipal Science and Technology Commission (No. Z161100000116047).

\section{Conflict of interest}

The authors have no conflict of interests related to this publication.

\section{Author contributions}

Conceived and designed the study ( $F L, S D)$, provided the original data $(\mathrm{LZ}, \mathrm{JZ}, \mathrm{WL})$, analyzed the results and wrote the manuscript ( $X W, M Y)$, provided assistance with data collection $(Y Z, X Q)$, reviewed and edited the manuscript $(Y L, J C, J Z$ and $C X)$, contributed equally to this paper (XW, MY).

\section{References}

[1] Torre LA, Bray F, Siegel RL, Ferlay J, Lortet-Tieulent J, Jemal A. Global cancer statistics, 2012. CA Cancer J Clin 2015;65:87-108. doi: 10.3322/caac. 21262.

[2] El-Serag HB, Rudolph KL. Hepatocellular carcinoma: epidemiology and molecular carcinogenesis. Gastroenterology 2007;132:2557-2576. doi: 10.1053/j.gastro.2007.04.061.

[3] Are C, Meyer B, Stack A, Ahmad H, Smith L, Qian B, et al. Global trends in the burden of liver cancer. J Surg Oncol 2017;115:591-602. doi: 10.1002/jso. 24518.

[4] Fong ZV, Tanabe KK. The clinical management of hepatocellular carcinoma in the United States, Europe, and Asia: a comprehensive and evidence-based comparison and review. Cancer 2014;120:2824-2838. doi: 10.1002/cncr. 28730.

[5] Hu XG, Mao W, Park YK, Xu WG, Kim BW, Wang HJ. Blood neutrophil-tolymphocyte ratio predicts tumor recurrence in patients with hepatocellular carcinoma within milan criteria after hepatectomy. Yonsei Med J 2016;57: 1115-1123. doi: 10.3349/ymj.2016.57.5.1115.

[6] Reichl P, Mikulits W. Accuracy of novel diagnostic biomarkers for hepatocellular carcinoma: An update for clinicians (Review). Oncol Rep 2016;36:613625. doi: 10.3892/or.2016.4842.

[7] EASL-EORTC clinical practice guidelines: management of hepatocellular carcinoma. J. Hepatol 2012;56:908-943. doi: 10.1016/j.jhep.2011.12.001.

[8] Yao M, Zhao J, Lu F. Alpha-fetoprotein still is a valuable diagnostic and prognosis predicting biomarker in hepatitis $B$ virus infection-related hepatocellular carcinoma. Oncotarget 2016;7:3702-3708. doi: 10.18632/oncotarget. 6913.

[9] Wen X, Lu F, Liu S. Prognostic value of p53 mutation for poor outcome of Asian primary liver cancer patients: evidence from a cohort study and metaanalysis of 988 patients. Onco Targets Ther 2016;9:7425-7433. doi: 10 . 2147/OTT.S121594.

[10] Xie X, Yao M, Chen X, Lu W, Lv Q, Wang K, et al. Reduced red blood cell count predicts poor survival after surgery in patients with primary liver cancer. Medicine (Baltimore) 2015;94:e577. doi: 10.1097/MD.0000000000000577.

[11] Heimbach JK, Kulik LM, Finn RS, Sirlin CB, Abecassis MM, Roberts LR, et al. AASLD guidelines for the treatment of hepatocellular carcinoma. Hepatology 2018;67:358-380. doi: 10.1002/hep.29086.

[12] Pugh RN, Murray-Lyon IM, Dawson JL, Pietroni MC, Williams R. Transection of the oesophagus for bleeding oesophageal varices. Br J Surg 1973;60:646649. doi: 10.1002/bjs.1800600817.

[13] Cholongitas E1, Papatheodoridis GV, Vangeli M, Terreni N, Patch D, Burroughs AK. Systematic review: The model for end-stage liver diseaseshould it replace Child-Pugh's classification for assessing prognosis in cirrhosis? Aliment Pharmacol Ther 2005;22:1079-1089. doi: 10.1111/j. 1365-2036.2005.02691.x.

[14] Poynard T, Bedossa P, Opolon P. Natural history of liver fibrosis progression in patients with chronic hepatitis C. The OBSVIRC, METAVIR, CLINIVIR, and DOSVIRC groups. Lancet 1997;349:825-832. doi: 10.1016/S0140-6736 (96)07642-8.

[15] Budczies J, Klauschen F, Sinn BV, Györffy B, Schmitt WD, Darb-Esfahani S, et al. Cutoff Finder: a comprehensive and straightforward Web application enabling rapid biomarker cutoff optimization. PLoS One 2012;7:e51862. doi: 10.1371 /journal.pone.0051862.

[16] The guidelines for the diagnosis and treatment of primary liver cancer (2017). Available from: http://www.nhfpc.gov.cn/yzygj/s7659/201706/ 80abf02a86c048fcb130e5e298f7aeee.shtml.

[17] Malinchoc M, Kamath PS, Gordon FD, Peine CJ, Rank J, ter Borg PC. A model to predict poor survival in patients undergoing transjugular intrahepatic portosystemic shunts. Hepatology 2000;31:864-871. doi: 10.1053/he.2000. 5852.

[18] Kamath PS, Wiesner RH, Malinchoc M, Kremers W, Therneau TM, Kosberg CL, et al. A model to predict survival in patients with end-stage liver disease. Hepatology 2001;33:464-470. doi: 10.1053/jhep.2001.22172.

[19] Fanali G, di Masi A, Trezza V, Marino M, Fasano M, Ascenzi P. Human serum albumin: from bench to bedside. Mol Aspects Med 2012;33:209-290. doi: 10.1016/j.mam.2011.12.002.

[20] Johnson PJ, Berhane S, Kagebayashi C, Satomura S, Teng M, Reeves HL, et al. Assessment of liver function in patients with hepatocellular carcinoma: a new evidence-based approach-the ALBI grade. J Clin Oncol 2015;33:550558. doi: $10.1200 /$ JCO.2014.57.9151.

[21] Ingenbleek $Y$, Young V. Transthyretin (prealbumin) in health and disease: nutritional implications. Annu Rev Nutr 1994;14:495-533. doi: 10 1146/annurev.nu.14.070194.002431.

[22] Zhao WC, Zhang HB, Yang N, Fu Y, Qian W, Chen BD, et al. Preoperative predictors of short-term survival after hepatectomy for multinodular hepatocellular carcinoma. World J Gastroenterol 2012;18:3272-3281. doi: 10. 3748/wjg.v18.i25.3272. 